\title{
Présentation : les discours du rituel
}

\section{Pierre Déléage}

\section{OpenEdition}

\section{Journals}

Édition électronique

URL : http://journals.openedition.org/jsa/11645

DOI : 10.4000/jsa. 11645

ISSN : 1957-7842

\section{Éditeur}

Société des américanistes

\section{Édition imprimée}

Date de publication : 5 octobre 2011

Pagination : 77-86

ISSN : 0037-9174

\section{Référence électronique}

Pierre Déléage, «Présentation : les discours du rituel », Journal de la société des américanistes [En ligne], 97-1 | 2011, mis en ligne le 10 juillet 2011, consulté le 22 avril 2019. URL : http:// journals.openedition.org/jsa/11645; DOI : 10.4000/jsa.11645 


\title{
PRÉSENTATION \\ LES DISCOURS DU RITUEL
}

\author{
Pierre DÉLÉAGE *
}

\begin{abstract}
I'm not particularly interested in "smoothed-over " versions of native culture. I like the stuff in the raw, as felt and dictated by the natives [...] the genuine, difficult, confusing, primary sources. These must be presented, whatever else is done [...]. There are too many glib monographs most of which time will show to be highly subjective performances. We need to develop in cultural anthropology that anxious respect for documentary evidence that is so familiar to the historian, and the classical scholar. Edward Sapir, correspondance de 1938 (cité in Foster 2005, p. 237)
\end{abstract}

En 1949, à l'occasion d'un essai synthétique sur le chamanisme destiné au Handbook of South American Indians, Alfred Métraux (1949, p. 583) remarqua que si l'on savait depuis longtemps que toutes les cérémonies rituelles de la région comportaient des incantations et des chants, il n'existait cependant aucun moyen de les étudier, faute de publication. Seul le recueil élaboré chez les Taulipáng et publié en 1923 par Theodor Koch-Grünberg faisait exception. Métraux dut donc se contenter d'inférer, à partir de la lecture de récits mythiques amazoniens, les principes et les entités qui auraient pu correspondre à ce qu'on lui avait demandé de décrire, à savoir une « religion ».

La mythologie des peuples d'Amazonie ${ }^{1}$ commençait alors depuis peu à être disponible dans les bibliothèques d'Europe et d'Amérique. Il vaut d'ailleurs peut-être la peine de rappeler qu'en dehors des courts extraits que l'on pouvait glaner, ça et là, dans la littérature des missionnaires et des explorateurs, l'entreprise de collecte, de traduction et de publication de ce genre de récits avait été commencée par un Amérindien, Maximiano José Roberto, d'ascendance partiellement tariana. Ce dernier disposait, pour mener à bien ce type de travail, d'indispensables compétences linguistiques en tukano, tariana et nheengatu; de plus, son village, Tarumãmiry, formait un point de convergence par lequel passaient de nombreux Amérindiens de la région du Vaupès. Dans ces conditions, il

* CNRS, Laboratoire d'anthropologie sociale, 52 rue du cardinal Lemoine, 75005 Paris [deleagepierre@hotmail.com].

Journal de la Société des Américanistes, 2011, 97-1, pp. 77-86. (C) Société des Américanistes. 
entama, à partir des années 1870 , une collecte de récits mythiques auprès de ses nombreux visiteurs, n'hésitant pas de temps à autre à accompagner des explorateurs de passage afin d'accroître encore ses connaissances. Il traduisit en nheengatu l'ensemble des textes recueillis, les collationna et les mit en ordre afin de soumettre au jugement des anciens une version qui puisse faire autorité, suivant ainsi une procédure qui devait peut-être moins à la méthodologie des études folkloriques qu'à celle, plus traditionnelle, de l'acquisition de prestige parmi les caciques (tuxáua) de la région. Autre fait notable, les produits de ses efforts ne furent jamais publiés sous son nom : on les retrouve, traduits en italien ou en portugais, dans des ouvrages signés par João Barbosa Rodrigues, Ermanno Stradelli ou Antonio Brandão de Amorim (Orjuela 1983, pp. 127-140 ; Sá 2002).

Malgré cette abondance, très relative, de récits mythiques publiés, on reste aujourd'hui surpris par le nombre restreint des sources auxquelles Claude LéviStrauss fit appel lorsqu'il s'engagea dans une vaste exploration des mythes amazoniens, peu après que le Handbook fut complété ${ }^{2}$. Et on comprend mieux la nécessité de sa stratégique transition aux cultures d'Amérique du Nord : il existait en effet dans cette région une longue et riche tradition de publication de mythes et de discours rituels. Comme dans le Vaupès, elle avait commencé à l'initiative d'Amérindiens : tout au long du XIX ${ }^{\mathrm{e}}$ siècle, les premiers ouvrages portant sur la mythologie de la région furent rédigés et souvent publiés par des auteurs tels que le Seneca Benjamin Williams, les Tuscarora David Cusick et Elias Johnson, les Ojibwa William Warren et George Copway, les Ottawa Francis Assikinack et Andrew Blackbird, ou encore le Penobscot Joseph Nicolar, le Wyandott Peter Dooyentate Clarke et le Potawatomi Simon Pokagon (Déléage 2009 ; Round 2010). Par la suite, l'importante entreprise de documentation des traditions orales des Indiens d'Amérique du Nord, entamée par le Bureau of American Ethnology et poursuivie par l'école de Franz Boas, s'appuya très largement sur des auteurs amérindiens afin d'élaborer les magnifiques corpus de récits mythiques et de discours rituels dont la richesse et la précision restent à ce jour inégalées ${ }^{3}$. Les Mythologiques, mais aussi les rares réflexions que Lévi-Strauss consacra aux discours rituels, ne pouvaient guère trouver un meilleur environnement afin de s'épanouir complètement.

Le fossé quantitatif et qualitatif qui séparait l'étude des traditions orales dans l'Amérique du Nord et dans les basses terres d'Amérique du Sud s'expliquait évidemment par des conditions historiques très hétérogènes - moins hétérogènes cependant que celles de la Mésoamérique ou des hautes terres d'Amérique du Sud où l'entrelacement de l'oral et de l'écrit date des premières décennies de la colonisation hispanique. Jusque dans les années 1960, l'Amazonie demeura de loin le least known continent de l'atlas ethnologique et les premières générations d'anthropologues professionnels qui partirent observer ces sociétés désiraient avant tout faire reconnaître l'originalité et les caractères propres de ces ensembles culturels alors méconnus en les affranchissant des modèles explicatifs importés 
d'autres régions du monde. Le dernier tiers du siècle passé donna ainsi lieu à une avalanche de travaux de qualité qui non seulement comblèrent d'immenses lacunes ethnographiques, mais élaborèrent aussi des problématiques qui prirent rapidement place à l'avant-garde des débats anthropologiques. S'il n'est pas question ici de retracer les enjeux de cette histoire récente, on remarquera qu'il ne serait pas trop difficile de montrer que la divergence entre les deux grandes modélisations de l'ontologie propre aux collectifs amazoniens trouve une part significative de ses origines dans les différences qui séparent les traditions orales à partir desquelles elles furent élaborées : il est ainsi très possible que l'animisme selon Philippe Descola soit aux chants anent des Achuar ce que le perspectivisme d'Eduardo Viveiros de Castro est aux chants rituels des meurtriers et des chamanes araweté.

Nonobstant, il faut bien avouer que le patient travail de transcription, de traduction et d'analyse des discours rituels resta marginal dans le principal courant des études amazoniennes tout au long de ces années. Souvent, ces traditions discursives ne faisaient l'objet que d'un bref article ou d'un court chapitre d'une monographie ; presque toujours, elles n'étaient évoquées qu'en passant, la voix originale du chamane ou du spécialiste rituel demeurant recouverte par celle de l'anthropologue. Parallèlement, le volume de traditions mythologiques amazoniennes publié s'accrût à une vitesse fulgurante. L'œuvre de Lévi-Strauss stimula clairement ces recueils, mais la perspective structuraliste conduisit souvent à se contenter des seules traductions des récits (sans leur transcription en langue vernaculaire), voire de simples résumés. Si le corpus est devenu immense, la qualité des éditions reste extrêmement inégale, et la réflexion comparatiste sur la mythologie amazonienne est aujourd'hui à peu près au point mort.

Edward Sapir avait beau déplorer la rareté des travaux ethnologiques fondés sur l'analyse détaillée de corpus de discours correctement transcrits (voir l'exergue de cette présentation), il n'en demeure pas moins que c'est aux ÉtatsUnis, et en grande partie grâce à lui, qu'il existe une tradition de recherche alliant expertise linguistique et perspective anthropologique. On ne s'étonnera donc pas que ce soit dans ce pays, pour l'essentiel à partir des années 1980, que s'est constitué un groupe d'anthropologues linguistes travaillant en Amazonie: ils s'attachèrent avant tout à l'étude de genres tels que les mythes, les récits " ordinaires » et l'art oratoire. Leur perspective était massivement ethnopoétique, leur permettant ainsi à la fois d'identifier les propriétés distinctives des différents genres discursifs et d'analyser avec précision les variétés du parallélisme, le dialogisme des récits, les modulations tonales des paroles chantées ou les usages indexicaux de certains modes d'expression. Parmi les membres de ce groupe qui écrivirent d'importantes monographies, on ne fera que mentionner Ellen Basso, Greg Urban, Laura Graham, Janet Hendricks, Janis Nuckolls ou Suzanne Oakdale, auxquels on joindra des anthropologues de formation plus 
classique, mais qui s'inspirèrent de leurs problématiques pour étudier des chants rituels tels qu'Anthony Seeger, Jonathan Hill, Robin Wright ou Dale Olsen ${ }^{4}$. En dehors des États-Unis, les recherches sur ces phénomènes discursifs restèrent le plus souvent l'apanage des linguistes et leurs publications demeurèrent en grande partie confinées à la revue Amerindia, ce qui en fait un véritable trésor pour tout anthropologue à la recherche d'analyses fines et précises des traditions orales amazoniennes ${ }^{5}$. Ce courant de recherche est toutefois resté marginal en Amazonie : trop rares encore sont les anthropologues qui font l'effort de publier les transcriptions et les traductions précises des discours à partir desquels ils formulent leurs spéculations et de très nombreuses traditions orales amazoniennes restent encore largement inconnues, faute d'enquête ${ }^{6}$.

À l'origine de ce dossier du Journal de la Société des Américanistes, un simple constat: un groupe de jeunes anthropologues travaillant en Amazonie, sur le point d'achever leur thèse de doctorat ou l'ayant tout juste défendue, avait pour caractéristique commune d'élaborer ses recherches à partir des difficult, confusing, primary sources qu'évoquait Sapir. Leur intérêt pour les pratiques discursives des sociétés qu'ils étudiaient passait par l'enregistrement, la transcription, l'analyse linguistique, la traduction et l'édition de ces discours oraux sous forme de textes; leurs lecteurs pouvaient ainsi évaluer leurs analyses en se rapportant à des sources dorénavant réinterprétables et améliorables à l'infini. Au cours de leurs enquêtes, tous avaient eu en particulier l'occasion d'éditer et d'étudier les discours rituels des sociétés qui les accueillirent - discours qui s'inscrivaient dans le cadre de cérémonies thérapeutiques, de fêtes de boisson, de rituels de chasse ou de liturgies propitiatoires. C'est donc autour de ces discours du rituel que s'est organisé ce dossier.

Chaque auteur a ensuite développé à sa guise sa propre approche théorique. D’une manière générale, il est vrai que tous, certains plus que d'autres, font appel aux principaux outils de l'anthropologie linguistique, ce qui leur permet de décrire précisément des phénomènes tels que le parallélisme, l'opacification des discours, le symbolisme sonore, l'indexicalité ou les relations formelles entre mythes et chants. Leurs problématiques d'ensemble s'élaborent néanmoins à partir d'horizons très variés : analyse interactionniste dans la lignée de Goffman, théorie critique, perspectivisme poétique, ethnolinguistique ou encore anthropologie pragmatique. C'est ainsi que, d'emblée, il est possible d'apercevoir un autre trait commun à l'ensemble de ces textes, trait qui n'avait pas été prévu : tous effectuent un saut immédiat de l'ethnographie linguistique la plus fine aux interprétations théoriques les plus générales, sans passer par la généralisation régionale en termes d'aires culturelles. Il ne faut pas voir là un quelconque anti-américanisme, mais plutôt une étape dans le déroulement de la recherche en Amazonie, les générations antérieures ayant assez profondément balisé le champ des spécificités sociologiques, ontologiques ou historiques des sociétés de la région. 
En dehors de ces aspects généraux, il me semble qu'une problématisation largement partagée constitue le fil conducteur de ce dossier. En effet, les analyses de discours rituels amazoniens qu' on y trouvera ne se limitent jamais à la recherche des éléments d'une métaphysique, ni à celle d'une clef de lecture de la réalité sociale, ni à une comparaison des structures poétiques des genres discursifs. Si l'ensemble de ces aspects est bien évidemment présent, il apparaît néanmoins subordonné à une recherche de ce que pensent les Amérindiens de leurs propres discours traditionnels. C'est pourquoi chaque auteur a éprouvé le besoin de procéder à une confrontation entre des registres discursifs hétérogènes d'une même société : afin de mettre au jour les propriétés réflexives qui, aux yeux des détenteurs de ces savoirs, permettaient de répartir leurs traditions discursives en domaines distincts. Cette approche réflexive se déploie tout au long de ce dossier en deux volets complémentaires : au nom de quelle autorité les discours rituels sont-ils énoncés et transmis ? qu'est-ce qui, au sein de ces discours, est pensé comme traditionnel? D'une certaine manière, les auteurs de ce dossier ont souhaité confier aux Amérindiens eux-mêmes la définition de ce sur quoi ils travaillaient. Cette prise en compte doit être comprise à partir de la situation historique nouvelle au cours de laquelle ces enquêtes se sont déroulées.

En Amazonie, les anthropologues commencent enfin à être confrontés à la crise d'autorité qui a secoué la plupart des ethnologies régionales depuis déjà un certain temps. Si les spécialistes des cultures d'Amérique du Nord ont depuis longtemps assimilé les virulentes critiques d'un Vine Deloria Jr., le problème ne s'est posé qu'assez récemment dans les sociétés amazoniennes, en partie en raison de rythmes différents dans les processus de scolarisation et d'accès à l'espace médiatique public. Cette crise d'autorité s'y est d'abord introduite par le biais de conflits concernant la biopiraterie et diverses formes de propriétés intellectuelles (Carneiro da Cunha 2010). Mais elle est aujourd'hui présente à l'esprit de tout anthropologue souhaitant étudier et publier des traditions orales : ses collaborateurs amérindiens ne tarderont souvent pas à soulever la question de la propriété intellectuelle de ces discours traditionnels, des modalités de leur publication, des noms qui doivent apparaître sur la couverture d'un livre ou sur la première page d'un article, de la répartition des droits d'auteur, de la teneur des interprétations que l'anthropologue va se permettre de proposer ou encore de la future « restitution" de son enquête à la communauté où elle a été effectuée. Ainsi, la question de l'autorité ou, si l'on veut, de l'auctorialité des discours traditionnels, est devenue pressante en Amazonie : parmi les indices de ce nouvel air $\mathrm{du}$ temps, on ne fera que mentionner la multiplication des publications, par les Amérindiens eux-mêmes, de versions de leurs propres traditions, suivant les exemples de Maximiano José Roberto et des écrivains iroquois ou algonquiens du XIX ${ }^{\mathrm{e}}$ siècle (Wright 2005 ; Hugh-Jones 2010) ou, encore, l'inflexion récente de l'anthropologie linguistique de la région vers la problématique de l'autobiographie (Oakdale 2007). 
Toute crise d'autorité est une bonne nouvelle : il ne doit pas être question d'en sortir, elle doit être choyée. C'est pourquoi il est heureux que de nombreuses alternatives fassent leur apparition: si les enquêtes issues du point de vue monologique d'un anthropologue ne doivent pas perdre leur légitimité, elles commencent à aménager une place pour les publications où l'anthropologue s'efface devant ses co-auteurs amérindiens ou pour celles où la relation dialogique est d'emblée clairement affichée (Kopenawa et Albert 2010 ${ }^{7}$ ). Il serait aujourd'hui impensable de publier les élaborations cosmologiques idiosyncrasiques et quasi ogotemmêliennes du Desana Antonio Guzmán sans que son nom apparaisse sur la couverture du livre et sans qu'il soit crédité, au moins en partie, de la propriété intellectuelle de l'œuvre (Reichel-Dolmatoff 1973). Une autre option, peut-être rendue possible par cette nouvelle crise d'autorité, consiste à effectuer un pas de côté et à problématiser les discours que tiennent les Amérindiens sur les auteurs de leurs traditions orales. Les discours traditionnels internalisent généralement cette problématique : dans la mesure où ils sont « hérités » d'une « tradition », leur auteur originel, garant de la légitimité de ces discours, est souvent pensé au croisement des frontières ontologiques, qu'il s'agisse des êtres des temps passés pour les récits mythiques ou d'entités surnaturelles pour les discours chamaniques.

On trouvera ces phénomènes divers d'attributions d'autorité étudiés dans toutes les contributions de ce dossier ; certains s'y attachent cependant plus que d'autres. À partir d'une approche dissolvante de la notion de ritualisation, qui ne peut être que bienvenue dans un dossier consacré aux discours rituels, Christopher Ball décrit de très brèves interactions cérémonielles wauja au cours desquelles de courts énoncés thérapeutiques sont échangés dans le cadre d'un dialogue entre un malade et des représentants d'esprits. L'étude détaillée de l'usage rituel des pronoms indexicaux de ces énoncés montre la complexité à la fois des positions d'autorité qui sont assumées par les participants et l'enchevêtrement performatif des relations de transformation et d'échange qui sont ainsi signifiées. Cédric Yvinec étudie les conséquences de l'attribution des chants rituels suruí à deux types d'auteurs distincts : les esprits chamaniques et les grands hommes. Elles apparaissent aux niveaux des contextes rituels de leur énonciation, de leurs modes d'apprentissage, de leur poétique ou encore de leurs effets perlocutoires. Andrea-Luz Gutierrez-Choquevilca analyse, quant à elle, deux genres de chants, cynégétiques et thérapeutiques, chez les Quechua du Pastaza en focalisant son attention sur la complexité des usages des onomatopées à l'intérieur de ces chants. En prenant en compte les modalités de l'apprentissage et de la transmission de ces incantations, elle montre que le symbolisme sonore joue un rôle comparable à celui des pronoms indexicaux dans le processus d'accumulation d'autorités surnaturelles distinctes qui forme l'enjeu principal de ces rituels.

Liées de près aux problèmes d'autorité, les réflexions amérindiennes sur la notion de «tradition » sont également devenues un enjeu majeur en Amazonie. 
Cela est particulièrement vrai au sein des sociétés dont les traditions tendent à s'effacer pour faire place à de nouveaux intérêts. Là aussi, pour les auteurs de ce dossier, il s'agit d'effectuer un pas de côté : délaisser la stérile opposition entre les approches essentialistes et relativistes de la tradition afin de prendre comme objet la notion de "tradition » que les Amérindiens formulent et utilisent. Pour ce faire, il est impossible de disjoindre deux questions complémentaires : qu'est-ce qui, dans une tradition amérindienne, est effectivement transmis ? qu'est-ce qui, dans l'ensemble de ce qui est transmis, est pensé par les Amérindiens comme « traditionnel»? Les anthropologues ne vont plus dans les sociétés amérindiennes avec, dans leurs bagages, leurs notions de culture, de tradition ou de rituel : ils attendent d'abord qu'on les leur fournisse. C'est alors seulement qu'il devient pertinent d'objectiver les traditions discursives amérindiennes : en considérant que ces phénomènes sont d'emblée réflexifs et qu'ils comportent leur propre définition. Plusieurs des auteurs de ce dossier ont par ailleurs été accueillis par des sociétés qui avaient indéniablement un problème avec leur culture traditionnelle, soit qu'elles la pensaient comme perdue, soit qu'elles la rejetaient avec virulence. Dans ces situations, leurs observateurs ne pouvaient guère que prêter attention à la manière dont la notion de « tradition » était problématisée par les Amérindiens eux-mêmes (Fausto s. d.). Cette réflexivité vis-à-vis des traditions et du traditionalisme n'est toutefois pas l'apanage des sociétés en transition culturelle : on la retrouve chez des peuples dont les discours rituels continuent à être transmis et elle forme un second horizon problématique que partagent, à des degrés divers, tous les auteurs de ce dossier.

Laurent Fontaine présente ainsi une longue invocation yucuna, employée dans de nombreuses incantations thérapeutiques, qui entrelace deux genres de discours : le premier est constitué de l'invocation elle-même, extrêmement formalisée, dont l'ordre séquentiel correspond à des trajets spatiaux formant une véritable cartographie de l'espace social et surnaturel des Yucuna; le second se compose des exégèses normatives de l'énonciateur sur sa propre invocation, commentaire réflexif, parfois d'ordre mythologique, qui, de manière surprenante, est lui aussi assez rigidement structuré. Cédric Yvinec prend comme point de départ des chants rituels qui, aujourd'hui, n'existent que dans la mémoire des Suruí, massivement convertis au protestantisme évangélique; ce faisant, il reconstruit les régimes réflexifs de la mémoire des traditions suruí autour des idées d'événement et de durée. Pedro de Niemeyer Cesarino élabore une réflexion sur les conditions de production et de transmission des différents genres de chants chamaniques marubo en restituant le système schématique de formules virtuelles à partir duquel ils sont produits, chacun avec sa propre configuration réflexive. Le processus de création perpétuelle au fondement de cette esthétique transcendantale ne se limite pas à la seule sémiotique discursive : il fonctionne également dans la production des danses cérémonielles et des innovations iconographiques des chamanes. Lucas Bessire part de la critique, par les Ayoréo, de la notion 
essentialiste de «tradition » qu'ont importée les diaboliques anthropologues; il poursuit en montrant que, ce qui se transmet dans le chamanisme ayoréo, ce sont moins des corpus de chants que des techniques schématiques de construction des incantations ujnarone à partir de données mythiques, traditionnelles ou chrétiennes. Les Ayoréo se sont en grande majorité convertis aux rituels chrétiens et à la communication par radio et Lucas Bessire propose de voir, dans ces derniers phénomènes, une adaptation de ces techniques de médiation temporelle à leur nouvel environnement. Emmanuel de Vienne étudie, quant à lui, la résurrection des chants rituels des Trumai qui envisagent leur « culture » sur le mode du manque et de la perte. À partir d'une reconstruction de l'histoire d'une grande cérémonie collective aujourd'hui disparue, il montre comment certains Trumai ont entièrement reconfiguré les propriétés réflexives de leurs traditions orales, transformant des compositions contextuelles en corpus rigides.

Espérons, au moment de clore cette brève présentation, que l'exigeant travail fourni par l'ensemble des auteurs de ce dossier contribuera à faire reconnaître la valeur, la complexité, l'intérêt et, parfois, la beauté des discours rituels amazoniens; et que sa lecture n'aurait pas déplu à Edward Sapir, le linguiste, l'anthropologue mais aussi le poète.

\section{Notes}

1. Dans cette présentation, le terme «Amazonie » est utilisé, pour des raisons de commodité, en référence à l'ensemble des basses terres d'Amérique du Sud.

2. D'autant plus que les œuvres les plus philologiques produites jusque-là, telles que celles de João Capistrano de Abreu ou de Konrad Theodor Preuss, étaient à peine prises en compte.

3. Sur certains de ces auteurs amérindiens, voir Liberty (1978). On trouvera quelques aperçus synthétiques de cette entreprise dans les premiers chapitres de Goddard (1996).

4. Voir aussi Sherzer et Urban (1986), Basso et Sherzer (1990) et Senft et Basso (2010).

5. Voir aussi Becquelin et Molinié (1993), Monod Becquelin et Erikson (2000), Monod Becquelin et Vapnarsky (2010).

6. Voir notamment Beier, Michael et Sherzer (2002). On trouvera dans cet article une liste assez exhaustive des travaux des linguistes et anthropologues que nous n'avons pas mentionnés ici. Les auteurs ont toutefois choisi d'écarter de leur recension les travaux des missionnaires, ce qui exclut certaines œuvres de grande qualité, telles que celles de Siro Pellizzaro ou de Cesareo Armellada.

7. Note de la rédaction: voir, dans ce volume, le riche compte rendu de cet ouvrage.

\section{RÉFÉRENCES CITÉES}

BAsso Ellen B. et Joel SHERzER (éd.)

1990 Las culturas nativas latinoamericanas a través de su discurso, Abya-Yala, Quito.

BECQUELIN Aurore et Antoinette Molinié (éd.)

1993 Mémoire de la tradition, avec le concours de Danièle Dehouve, Société d'ethnologie, Nanterre. 
BeIER Christine, Lev Michael et Joel Sherzer

2002 «Discourse forms and processes in Indigenous Lowland South America : an areal-typological perspective », Annual Review of Anthropology, 31, pp. 121-145.

Carneiro Da Cunha Manuela

2010 Savoir traditionnel, droits intellectuels et dialectique de la culture, Éditions de l'Éclat, Paris.

DÉLÉAGE Pierre

2009 «Les Amérindiens et l'écriture », L’Homme, 190, pp. 191-198.

Fausto Carlos

s. d. "Mil años de transformación en Amazonía : Los kuikuro entre el pasado y el futuro » [à paraître].

FOSTER Michael K.

2005 " Jacob Ezra Thomas : Educator and Conservator of Iroquois Culture », Histories of Anthropology Annual, 1, pp. 219-245.

GoDDARD Ives (éd.)

1996 Handbook of North American Indians. 17 : Languages, William C. Sturtevant general éd., Smithsonian Institution, Washington, DC.

Hugh-Jones Stephen

2010 «Entre l'image et l'écrit: la politique tukano de patrimonialisation en Amazonie », Cahiers des Amériques Latines, 63-64, pp. 195-228.

Kopenawa Davi et Bruce AlberT

2010 La chute du ciel. Paroles d'un chaman yanomami, Plon, Paris.

LIBERTY Margot (éd.)

1978 American Indian intellectuals, West Publishing Co., St Paul.

MÉTRAux Alfred

1949 «Religion and shamanism », in Handbook of South American Indians. 5, Smithsonian Institution, Bureau of American Ethnology, Bulletin 143, Washington, DC, pp. 559-599.

Monod BeCQuelin Aurore et Philippe ERIKson (éd.)

2000 Les rituels du dialogue, Société d'ethnologie, Nanterre.

Monod Becquelin Aurore et Valentina VAPNARsky (éd.)

2010 "L'agentivité : ethnologie et linguistique à la poursuite du sens », Ateliers $d u$ LESC, 34 [http://ateliers.revues.org/8515, consulté le 02/10/2010].

OAKDALE Suzanne (éd.)

2007 "Special section : life history ", Tipití. Journal of the Society for the Anthropology of Lowland South America, 5 (1), pp. 1-95.

ORJUEla Héctor H.

1983 Yurupary : mito, leyenda y epopeya del Vaupés, Instituto Caro y Cuervo, Bogota. 


\section{ReICHEL-Dolmatoff Gerardo}

1973 Desana. Le symbolisme universel des Indiens tukano du Vaupès, Gallimard, Paris.

Round Philip H.

2010 Removable type: histories of the book in Indian country, 1663-1880, The University of Carolina Press, Chapel Hill.

SÁ Lúcia

2002 «A Lenda do Jurupari : texto sagrado ou fruto da imaginação de littérateurs? », in Sérgio Medeiros (éd.), Makunaíma e Jurupari : cosmogonias ameríndias, Editora Perspectiva, São Paulo, pp. 347-358.

SENFT Gunter et Ellen B. Basso (éd.)

2010 Ritual communication, Berg Publishers, Oxford.

SHERZER Joel et Greg Urban (éd.)

1986 Native South American discourse, Mouton de Gryuter, Berlin.

WRIGHT Robin

2005 «Da atribuição de valores às tradições religiosas indígenas », Revista do Patrimônio Histórico e Artístico Nacional, 32, pp. 244-253. 